\title{
Experimental validation of a model-based method for separating the space heating and domestic hot water components from smart-meter consumption data
}

\author{
Rasmus Elbak Hedegaard ${ }^{1, *}$, Martin Heine Kristensen ${ }^{1,2}$, and Steffen Petersen ${ }^{1}$ \\ ${ }^{1}$ Aarhus University, Inge Lehmanns Gade 10, 8000 Aarhus C, Denmark \\ ${ }^{2}$ AffaldVarme Aarhus (Department of Waste and District Heating), Karen Blixens Boulevard 7, 8220 Brabrand, Denmark
}

\begin{abstract}
Smart meters are currently being rolled out in European district heating (DH) systems at a large scale to enable time-varying district heating tariffs and improve consumer awareness about their own consumption. Smart-meter data can also be used in more advanced applications, e.g. for establishing modelbased control schemes for demand response purposes and data-driven building energy performance labeling schemes. Many of these applications require separate measurements of the consumption for space heating (SH) and preparation of domestic hot water (DHW); however, smart meters often only provide the total DH energy consumption (SH+DHW) in truncated units (e.g. whole kWh on an hourly basis). Typical approaches for separating these two components of DH consumption require measurements with a high temporal and numerical resolution and are therefore not applicable to smart-meter data. New methods suitable for disaggregating the combined DH demand are therefore needed. This paper presents a validation of a modelbased method for disaggregating DH consumption using ground truth data from 44 residential buildings.
\end{abstract}

\section{Introduction}

In an effort to improve the transparency and billability of energy consumption, a recent amendment to the energy efficiency directive (EED) of the European Union (EU) introduced a requirement for all newly installed district heating $(\mathrm{DH})$ and cooling meters to be remotely readable by 25 October 2020, while all remaining non-remotely readable meters is to be replaced by 1 January 2027 [1]. Modern remotely read heat meters are capable of reporting consumption at an hourly or sub-hourlytemporal resolution, which is a significant leap forward compared to the annual temporal resolution that is often associated with manually read meters. The improved availability of high-resolution smart-meter consumption data is one of the cornerstones in enabling several new technologies to be implemented in building automation. One of these technologies is model-based control schemes for heating systems, which may improve the comfort of occupants while minimizing energy consumption by optimizing HVAC control [2,3]. These control schemes also allow for utilizing the thermal inertia of buildings to render part of the consumption in the buildings flexible, thereby enabling building owners to sell services to the heating utility. The models used to establish such control schemes need to describe the thermal dynamic behavior of the buildings accurately and are therefore typically obtained by calibrating the parameters of said models using measurements of internal temperatures, external weather conditions, and heating consumption. Since the latter of these measurements is often the hardest to obtain, the availability of data from already installed smart meters may be a decisive factor for the viability of such control schemes. Another promising use of high-quality consumption data is for generation of data-driven building energy performance certificates as a supplement or even an alternative to traditional energy audits. However, the applications outlined here require separate measurements of the energy consumed for space heating ( $\mathrm{SH})$; currently, DH smart meter measurements is a sum of the energy used for SH and for preparation of domestic hot water (DHW). Therefore, previous studies have investigated inferencebased techniques for disaggregating district heating measurements to estimate the energy quantities consumed for each of these two purposes. Two prevailing approaches have been identified, which we shall refer to as the summer methods and the inertia methods, respectively. A third approach, which is not further discussed in this study due to a lack of water consumption data, relies on measurements of the cold water consumption together with the assumption that a certain fraction of the consumed water is heated before use.

The summer methods are based on the assumption that all DH consumption during the warmest period of the year can be attributed to DHW production [4,5], and that this is representative for the entire year. The period for which this assumption is reasonable depends of course on the climate that the building is situated in. Therefore, some implementations of the summer method attempt to account for space heating through various assumptions.

* Corresponding author: reh@eng.au.dk 
The advantage of the summer methods is that they are straightforward to implement and can be applied to time series within a wide range of temporal resolutions (minutes to months). A disadvantage of the summer methods is the uncertainty related to the presence and magnitude of any weather-independent space heating in summer time (e.g. bathroom underfloor heating). The perhaps largest flaw of the summer methods is that they rely on measurements from a period where many people are home from work or school, or on holiday. In many EU countries, it seems likely that the building occupation and DHW consumption during summertime is actually the least representative for the whole year.

Instead of relying on warm weather to isolate DHW consumption, the inertia methods rely on the differences between the relatively smooth characteristics of space heating consumption and the more volatile and rapidly varying DHW consumption to disaggregate the total consumption. While inertia methods may be implemented in several ways, their common ground is that they attempt to detect DHW consumption from peaks in the consumption profile, which are considered unlikely to be caused by the space heating demand [6,7]. Once detected, these peaks are subtracted from the overall consumption profile to arrive at separate estimates of DHW and space heating. Since this approach is not restricted to summertime measurements, but may use measurements from the heating season as well, the inertia methods avoid the main issues that apply to the summer methods. However, the advantages of the inertia methods come at the significant cost of the methods requiring measurements of high resolution to enable reliable and accurate detection of DHW consumption peaks of various magnitudes and durations; Yliniemi et al. [6] considered sampling rates lower than 60 seconds, while Bacher et al. [7] used 10-minute measurements. However, current DH smart meters setups does not deliver data in this resolution. Instead, they work by continuously aggregating consumption resulting in a monotonically increasing aggregate of consumption reported in whole $\mathrm{kWh}$ or MWh to lower memory usage. The heating utility can then remotely read-off the current aggregated consumption level at any desired frequency, for example at an hourly, monthly or even annual rate, depending on their data quality requirements and the available bandwidth on the wireless network that facilitates the read-off. A stationary time series of consumption levels with a resolution defined by the logging frequency, for instance $\mathrm{kWh} / \mathrm{h}$, may then be derived as the first-order difference of aggregated measurements. This type of data poses a major challenge for the inertia methods as even significant DHW-consuming activities may have a limited impact on the time series once converted to a truncated hourly temporal resolution. An example is a 5-minute shower, which, assuming a $45^{\circ} \mathrm{C}$ delivery temperature and $10^{\circ} \mathrm{C}$ mains temperature, amounts to approximately 1.6 $\mathrm{kWh}$ [8]. This consumption of $1.6 \mathrm{kWh}$ could result in the aggregated whole $\mathrm{kWh}$-unit value in the meter increasing by either 1 or $2 \mathrm{kWh}$, depending on the hidden decimals of the current aggregated value. As a result, part of the consumption may be shifted up to several hours in time, depending on the flow through the meter. Furthermore, since $1 \mathrm{kWh}$ is the lowest quantity of heat that is reported by the meter, such a peak could just as easily have been generated by space heating, thus making the inertia method highly unreliable for this type of data.
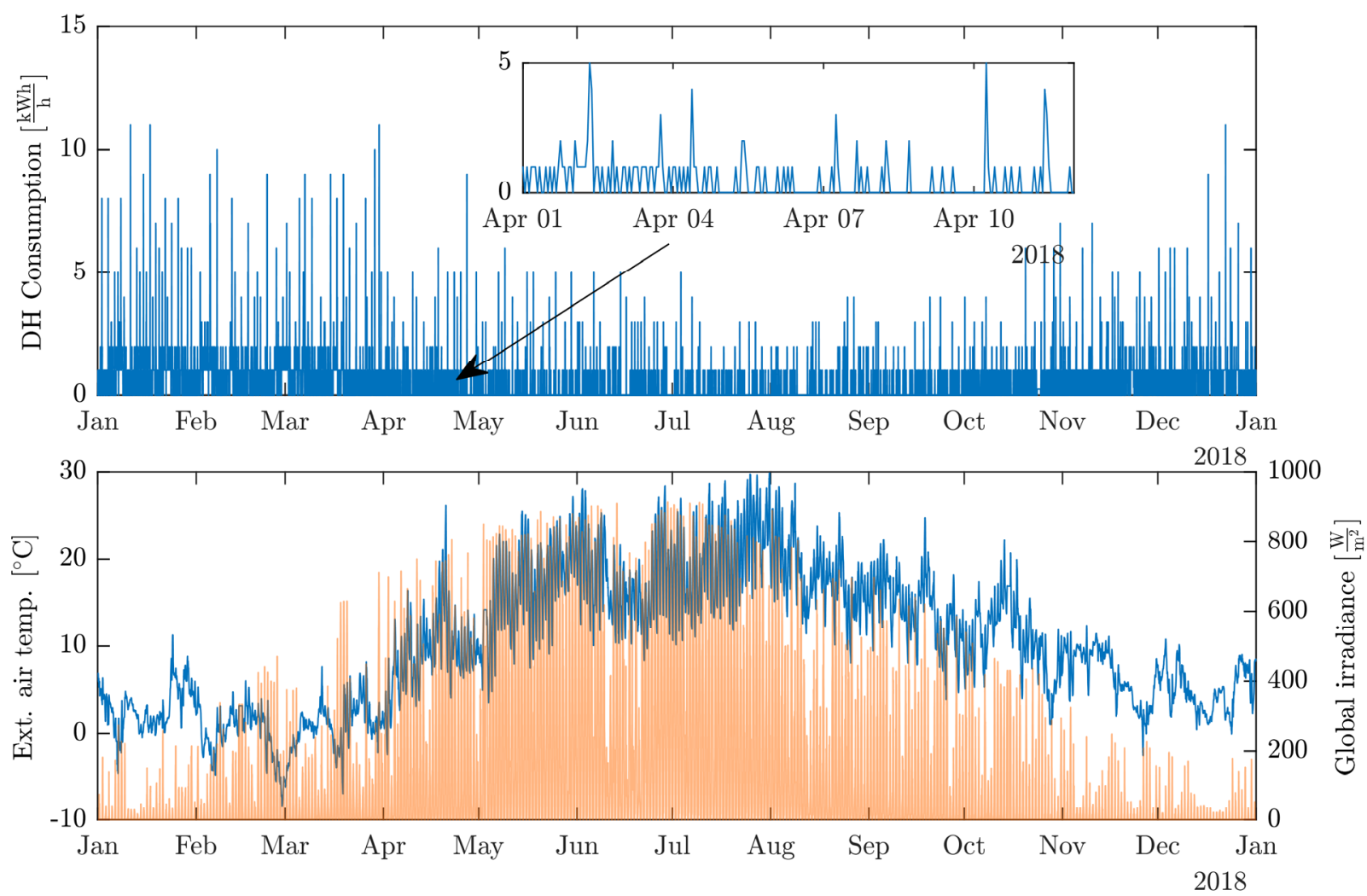

Fig. 1. The data used for the case study. Top: An example of a district heating consumption time series from a Kamstrup MULTICAL 602 energy meter. Bottom: Weather conditions (temperature, solar) for the period. 


\subsection{Scope of paper}

The described challenges of the summer and inertia methods call for the development of new approaches that are less vulnerable to seasonal effects and data resolution. In this paper, we present a validation of a method recently proposed by Hedegaard et al. [9] in which total district heating measurements from a building are disaggregated into SH and DHW, respectively, through parallel calibration of a model of the thermal dynamics of the building and a model of the domestic hot water consumption. The method, which we for the remainder of this paper shall refer to as the model-based method, was originally developed to facilitate data-driven energy modeling of residential buildings relying on smart-meter data, but it could also be applied for other purposes where $\mathrm{SH}$ and DHW needs to be separated. In the present paper, we apply the model-based method to 44 terraced singlefamily houses for which the utility company AffaldVarme Aarhus (Denmark) have provided hourly smart-meter measurements of the total district heating consumption, as well as separate measurements of district heating consumption for production of DHW. The latter enables us to evaluate the accuracy of the DHW consumption estimates obtained with the proposed model-based method as well as the summer method. Figure 1 depicts measurements of weather conditions together with the district heating consumption of one of the 44 terraced houses. The zoom-in on a part of the consumption time series demonstrates the truncation that characterizes the data. In addition, we develop the method further by evaluating the effectiveness of several proposed modifications. The following section describes the method and modifications before Section 3 presents the results of the validation and development efforts of the analysis. Finally, we discuss the findings and draw conclusions in Sections 4 and 5.

\section{Method}

The model-based method is conceptually simple in that it aims to establish separate models of the space heating and domestic hot water components of the measured district heating consumption. The field of system identification provides a large set of methods designed to identify the most likely set of parameter values that enable a given model to reproduce the measured input-output behavior of the modeled process. The premise of the model-based method is that such methods, when combined with suitable model structures for SH and DHW consumption, can be used to separate these two consumption components by identifying the set of parameters of each of the two respective models that best reproduce the combined time series of district heating consumption obtained from the smart meter. The following sections briefly introduce the models of $\mathrm{SH}$ and DHW consumption together with the statistical framework used for parameter identification in this implementation of the model-based method. For further details on the proposed method, we refer readers to the original paper [9].

\subsection{Space heating model}

Several past studies have used reduced-order models to represent the thermal dynamics of buildings in a variety of contexts including forecasting of temperature conditions [10-12], data-driven investigations of thermal properties [13-15] and in implementations of modelbased control schemes such as model predictive control [16-18]. Researchers typically choose either physicsbased grey-box models or their black-box counterparts characterized by their highly flexible but physically uninterpretable structure. Since each modeling approach has its own merits, it is typically the intended application of the models, and the availability and quality of measurement data, that determines which of the approaches is the most suitable. The model-based method was originally developed with the intent of establishing realistically behaving models of the thermal dynamics of buildings. With this in mind, the grey-box approach was adopted since it improves the ability of the modeler to evaluate the realism of the models and restrict parameter values to lie within what is considered physically meaningful.

The grey-box model used to represent the space heating component is a $5 \mathrm{R} 2 \mathrm{C}$ resistance-capacitance model. The model structure is an extension of the simple hourly method presented in ISO 13790:2008. While many other grey-box model structures are fully capable of representing the thermal dynamics of buildings, this particular grey-box model structure was chosen due to the high degree of physical interpretability of its parameters, which allowed us to use basic and publicly available building-related metadata and experiential (empirical) values to specify many of the building-related parameters prior to the calibration. The building metadata, which was obtained from the Danish Buildings and Dwellings Register (BDR), included the year of construction and heated floor area of the building. Eq. (1) lists the inputs used for establishing the building energy model that represents the demand for space heating, $\boldsymbol{\Phi}_{\mathrm{SH}}$, in a given building.

$$
\Phi_{S H, t}=F\left(\boldsymbol{x}, \boldsymbol{\theta}_{S H}, \boldsymbol{W}, t\right)
$$

Here, the vector $\boldsymbol{x}$ contains the fixed parameters of the thermal model that are not inferred from measurement data, while the vector $\boldsymbol{\theta}_{\mathrm{SH}}$ contains all the inferred parameters related to the building. The set of calibration parameters remains the same as in [9] and includes the 1) window-to-floor ratio, 2) infiltration rate, 3) average envelope U-value, 4) number of occupants, and 5) the effective thermal capacity of heavy building components. The number of occupants, $\theta_{\text {occ }}$, is the only one of these parameters that are also used in the DHW model. Finally, the matrix $\boldsymbol{W}$ contains relevant weather measurements for the measurement period while the time step is denoted $t$. 


\subsection{DHW model}

The choice of a model structure for the DHW consumption was based on the following three assumptions: 1) Any dynamic properties of DHW consumption may be neglected as consumption is nearinstantaneous, 2) DHW consumption can be considered to follow a repeating daily or weekly pattern that features a stochastic component, and 3) DHW consumption is largely weather-independent. Previous research studies have identified the number of occupants in a household as the best predictor of the overall DHW consumption in a dwelling [19,20], and have also found significant differences between the DHW consumption during working days and weekends [19]. Based on this, a simple switching model featuring 24-hour tapping profiles defining the distribution of DHW consumption within each day was formulated. Eq. (2) lists the inputs used in the DHW model to predict the DHW consumption in a given hour $t$.

$$
\Phi_{D H W, t}=F\left(\boldsymbol{\theta}_{D H W}, d_{t}, h r_{t}, E_{D H W}\right)
$$

The two tapping profiles of the model are stored as column vectors in the matrix $\boldsymbol{\theta}_{\mathrm{DHW}}$. The switching parameter $d$ refers to the type of day and defines which of the 24-hour profiles (column) that are active for a given day, while the hour-number $h r$ specifies which hour of the day (row in $\boldsymbol{\theta}_{\mathrm{DHW}}$ ) the current time step $t$ belongs to. Finally, $E_{\mathrm{DHW}}$ is the estimated annual consumption in the dwelling as defined by Eq. (3).

$$
E_{\text {DHW }}=\frac{c_{\text {p,water }} \rho_{\text {water }} V_{\text {occ }} \theta_{\text {occ }}\left(T_{\text {DHW }}-T_{\text {mains }}\right)}{365}
$$

Where $c_{\mathrm{p} \text {,water }}$ and $\rho_{\text {water }}$ are material properties of water, $V_{\text {occ }}$ is the average annual consumption of DHW per occupant and $\theta_{\text {occ }}$ is the inferred number of occupants in the building. Since the number of occupants and the consumption per occupant both appear as linear factors in Eq. (3), it is not feasible to calibrate both of them. Due to the number of occupants in each building being unknown, we choose to calibrate this parameter and fix the average DHW consumption per capita in Denmark, $V_{\text {occ }}$, to $15 \mathrm{~m}^{3}$ per year based on a report from the Danish Building Research Institute [21]. Finally, the delivery temperature of the DHW, $T_{\text {DHW }}$, is assumed $55^{\circ} \mathrm{C}$ and the mains water temperature, $T_{\text {mains }}$, is assumed to be $10{ }^{\circ} \mathrm{C}$.

\subsection{Statistical framework}

The parameters of the two models were identified using the maximum likelihood approach in which we assume that the measured consumption data can be regarded as the sum of an energy-consuming process $\boldsymbol{\Phi}_{\text {sim }}$ and a normally distributed stochastic component $\boldsymbol{\epsilon}$ with zero mean. The energy-consuming process consists of two separate components, namely the space heating component, $\boldsymbol{\Phi}_{\mathrm{SH}}$, and the domestic hot water component, $\boldsymbol{\Phi}_{\mathrm{DHW}}$. The assumed relationship between the smartmeter measurements and the output of the models is described in Eq. (4)-(6), in which index $t$ refers to the hour of measurement.

$$
\begin{gathered}
\Phi_{D H, t}=\Phi_{s i m, t}+\epsilon_{t} \\
\Phi_{s i m, t}=\Phi_{S H, t}+\Phi_{D H W, t} \\
\epsilon_{t} \sim N\left(0, \sigma^{2}\right)
\end{gathered}
$$

The parameters of the models were calibrated by maximizing the likelihood of the data conditional on the calibrated parameters of the two models and the stochastic component of Eq. (6), respectively. The likelihood function is defined by Eq. (7), where $\sigma^{2}$ is the variance of the stochastic component and $\boldsymbol{\epsilon}$ is the error between the combined model output and the measured consumption.

$$
p\left(\boldsymbol{\Phi}_{D H} \mid \boldsymbol{\theta}_{S H}, \boldsymbol{\theta}_{D H W}, \sigma\right)=\frac{1}{\sqrt{2 \pi \sigma^{2}}} e^{-\frac{1}{2 \sigma^{2}} \sum_{t=1}^{n_{t}} \epsilon_{t}^{2}}
$$

The physical interpretability of the model parameters furthermore allowed us to specify the prior probability of the calibrated model parameters as probability distributions reflecting our prior beliefs about the likely value of each parameter. Using priors to guide a parameter calibration process is useful whenever the input-output data used for the calibration does not contain the necessary information to identify all model parameters. To incorporate these a priori beliefs, we augment the likelihood function by introducing the priors in the new objective function in Eq. (8), which according to Bayes' theorem is proportional to the a posteriori likelihood of the parameters conditional on the data.

$$
\begin{gathered}
p\left(\boldsymbol{\theta}_{S H}, \boldsymbol{\theta}_{D H W}, \sigma \mid \boldsymbol{\Phi}_{D H}\right) \propto p\left(\boldsymbol{\Phi}_{D H} \mid \boldsymbol{\theta}_{S H}, \boldsymbol{\theta}_{D H W}, \sigma\right) \\
\cdot p\left(\boldsymbol{\theta}_{S H}\right) \cdot p\left(\boldsymbol{\theta}_{D H W}\right) \cdot p(\sigma)
\end{gathered}
$$

The prior distributions for the building-related parameters were based on findings from experimental studies and past building regulations, whereas the prior distributions for the parameters describing the DHW profiles were based on a measuring study mapping residential DHW consumption in the UK [20]. The DHW prior was slightly modified for the present analysis by increasing the probability of no DHW consumption during the first four hours of a day (00:00 - 04:00). This was done to reflect that around $90 \%$ of Europeans spend this part of night sleeping [22].

\subsection{Modifications}

The access to the separate measurements of DH consumption for production of DHW enables us to investigate the accuracy of the proposed model-based method, but it also enables us to investigate and validate whether the precision of the method can be improved by changing the way that the DHW model partitions the days of the week into archetypical tapping profiles. To this end, the four partitioning schemes presented in Table 1 were selected for evaluation. Each scheme is denoted by the number of unique tapping profiles employed in it, while letters A-G are used to indicate recurring use of a profile. The partitioning schemes with 1 and 7 profiles act as the 
two extremes in which all weekdays are considered as either identical or unique, respectively.

Table 1 Partitioning schemes of weekdays.

\begin{tabular}{|l|lllllll|}
\hline Partitioning & \multicolumn{7}{|c|}{ Weekday } \\
scheme & Mon & Tue & Wed & Thu & Fri & Sat & Sun \\
\hline 1 profile & A & A & A & A & A & A & A \\
2 profiles & A & A & A & A & A & B & B \\
4 profiles & A & A & A & A & B & C & D \\
7 profiles & A & B & C & D & E & F & G \\
\hline
\end{tabular}

The 2-profile scheme reflects a distinction between working days and weekend days. Finally, the 4-profile scheme reflects a partition of weekdays depending on both the working status of the current day and the day following it. For all partitioning schemes, the originally employed assumption of the daily DHW quantity being identical for all days across the year was relaxed, such that only days associated with a given profile are assumed to carry the same DHW quantity.

\section{Results}

Fig. 2 shows the ability of the method to estimate the DHW consumption patterns for the average week of 2018 from the truncated DH consumption data and how the choice of partitioning scheme affects performance. The compared time series are the aggregated modelled or measured consumption of all case buildings. Overall, the results show that the model-based method is capable of identifying the DHW consumption patterns with reasonable accuracy. It is also evident how the choice of partitioning scheme affects the estimates. As indicated by the normalized mean bias error (NMBE) depicted on each plot, the method is seen to overestimate the overall DHW quantity by approximately $13 \%$ regardless of the applied partitioning scheme. A possible reason for this discrepancy is the simplifications employed in the space heating model, which may result in some of the space heating consumption being absorbed by the DHW model.

A comparison of the time series reveals a poor performance of the 1-profile partitioning scheme, which approximates an average between working days and weekdays and as a result fails to describe neither of them particularly well. The 2-profile scheme shows a significant improvement for both the size and placement of morning peaks, while the evening peaks on Fridays and Saturdays are estimated inaccurately. The time series suggests that the size of the evening peak is dependent on whether occupants have work/school on the following day - perhaps due to some occupants showering in the evenings. The 4-profile scheme remedies this by treating each of the last three days of the week separately. The 7-profile scheme did not result in any significant improvement over the 4-profile scheme, and is even seen to result in an increase in the mean absolute error (MAE) for the average week profiles. A similar result is seen in Fig. 3, which presents the daily building-average MAE for all 52 full weeks of the year 2018. Because of these findings, the 4-profile partitioning scheme was adopted going forward. However, it should be noted that more
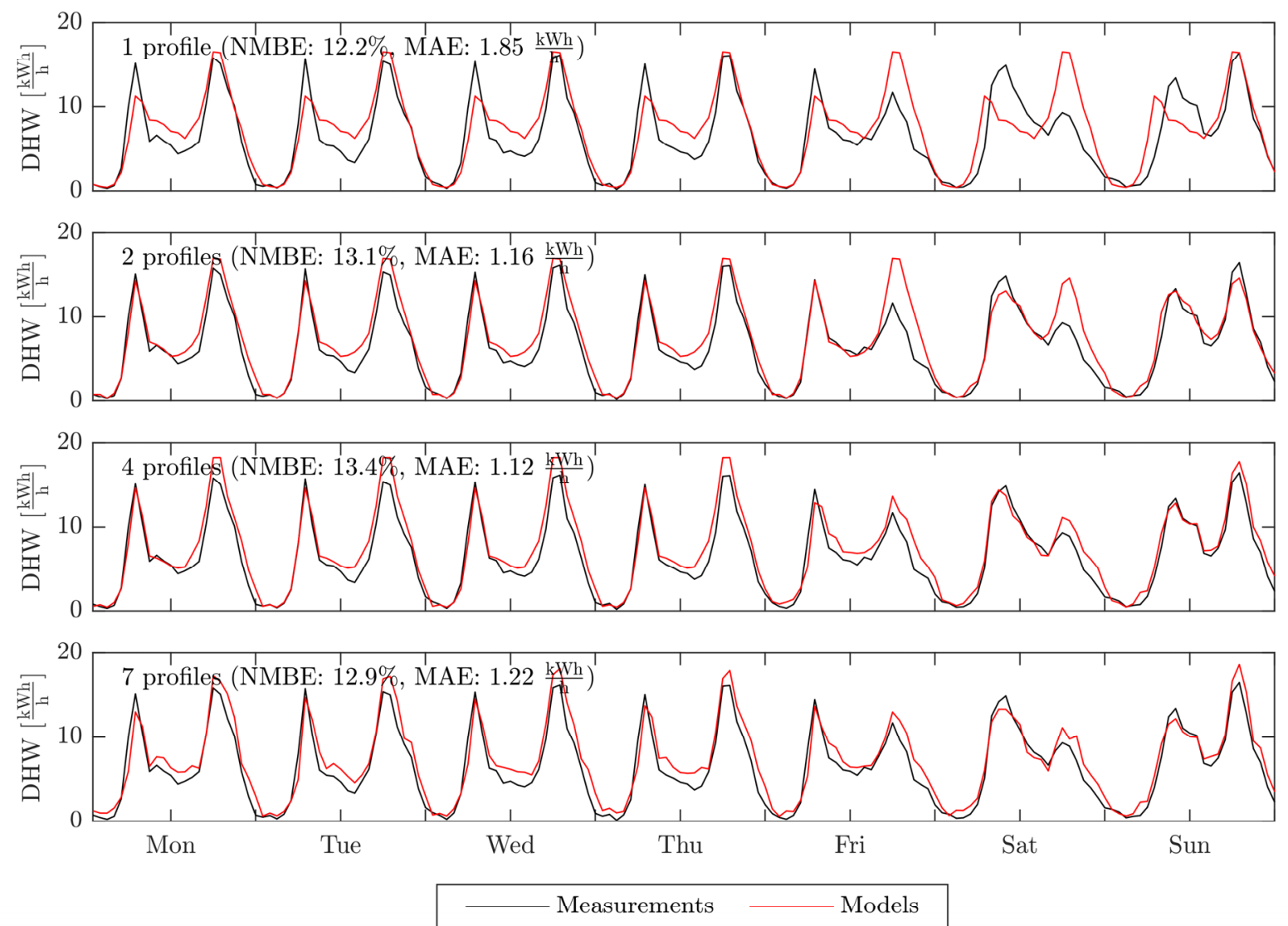

Fig. 2. Comparison of the modelled and measured week-profile averaged across all full weeks in the calendar year 2018 and aggregated across all of the 44 case buildings. 


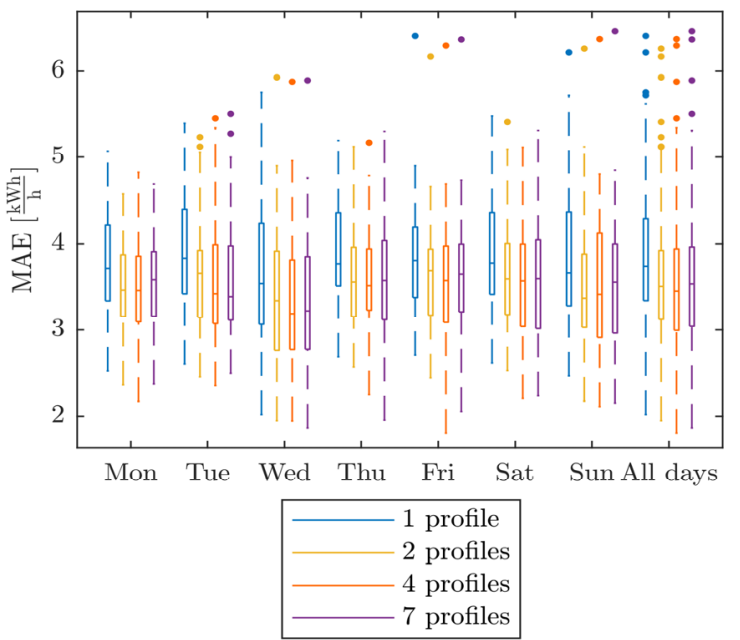

Fig. 3. MAE for DHW estimates for the aggregated 44 buildings distributed on week-days. Each day-specific boxplot contains 52 data points - one for each week of the year.

validation data is required to investigate the generality of this result. While Fig. 2 and 3 indicate the performance of the method when applied to a group of buildings, it is also interesting to evaluate the performance on the scale of individual buildings. Since it is impractical to depict the modeled profiles for all 44 buildings, we show six selected buildings (A-F) that are considered representative of the entire sample. The depicted buildings were selected by sorting the 44 buildings according to the mean absolute percentage error (MAPE) and then selecting an evenly spaced set of buildings from that list. The MAPE metric was preferred over the MAE since the latter tends to favor buildings with low consumption levels and thus may bias the comparison. This is evident from Fig. 4, which shows that increasing consumption levels tend to result in an increase in MAE but a decrease in MAPE. The latter indicates an improved accuracy of the disaggregation per unit of consumption in households with generally higher consumption levels.

Fig. 5 depicts a time series of the average week for each of the buildings highlighted in Fig. 4; the estimate of Building $\mathrm{A}$ and $\mathrm{F}$ are characterized by the worst and best MAPE values, respectively.

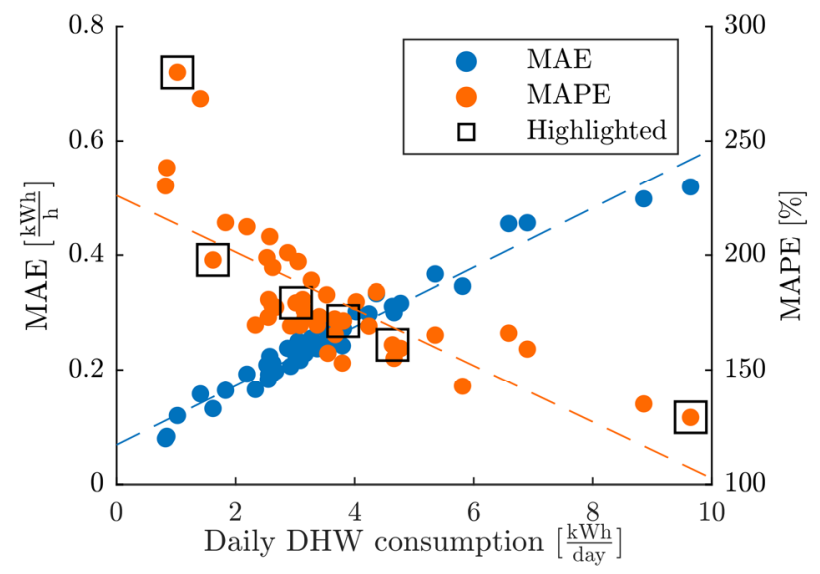

Fig. 4 Mean absolute error (MAE) and normalized MAE (MAPE) for each building. Square symbols on the MAPE data series indicate which buildings are depicted in Fig. 5, where the squares from left to right refers to buildings A-F.

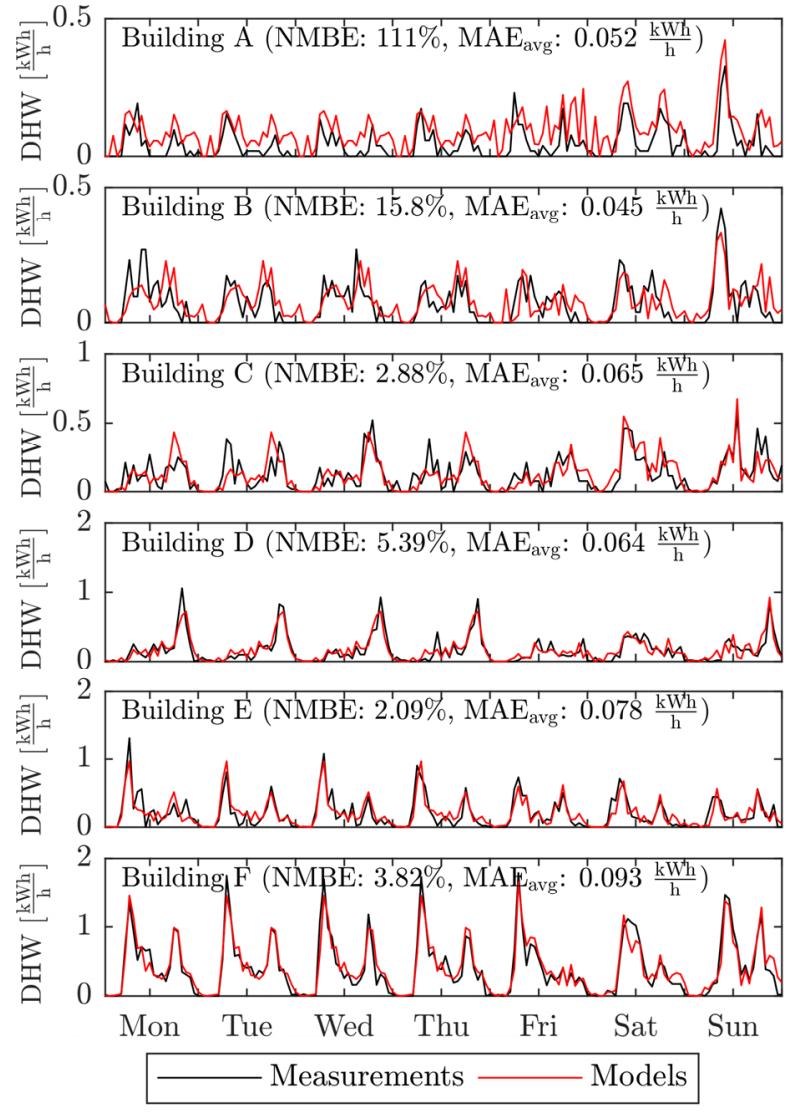

Fig. 5. Comparison of the measured and modelled DHW consumption levels (4-profile) for six buildings (A-F) that are considered representative of the group of 44 case buildings. The data depicts the average week in the calendar year of 2018.

The results in Fig. 5 indicate that the method also performs well on the scale of individual buildings, as it is capable of identifying the average week-profiles with generally high accuracy with MAE for the average week below $0.1 \frac{\mathrm{kWh}}{\mathrm{h}}$. It is important to stress that the stochastic nature of occupants and their DHW consumption results in the consumption patterns on individual weeks may deviate significantly from the profile of the average week depicted here. This is evident by comparing the MAE values indicated on Fig. 4, which are computed for all weeks to the average values of the week $\left(\mathrm{MAE}_{\mathrm{avg}}\right)$ in Fig. 5. This stochasticity is important to consider if the output of the DHW models are compared to individual weeks or applied for forecasting purposes. However, we consider it reasonable to assume that the majority of this stochasticity cancels out as the number of forecasted buildings increase. The same cannot be said if the DHW consumption estimated by the method tends to be biased across all buildings, as indicated by the NMBE values depicted in Fig. 2. The bias of the estimated amount of DHW consumption in each building is therefore presented in Fig. 6. For reference, the figure also depicts the estimates one would obtain when applying the summer method. To avoid choices in our implementation of the summer method influencing this comparison, we present a bias-free estimate of the consumption levels during summertime. The figure presents the results in both relative and absolute terms. The reason for doing so is that 

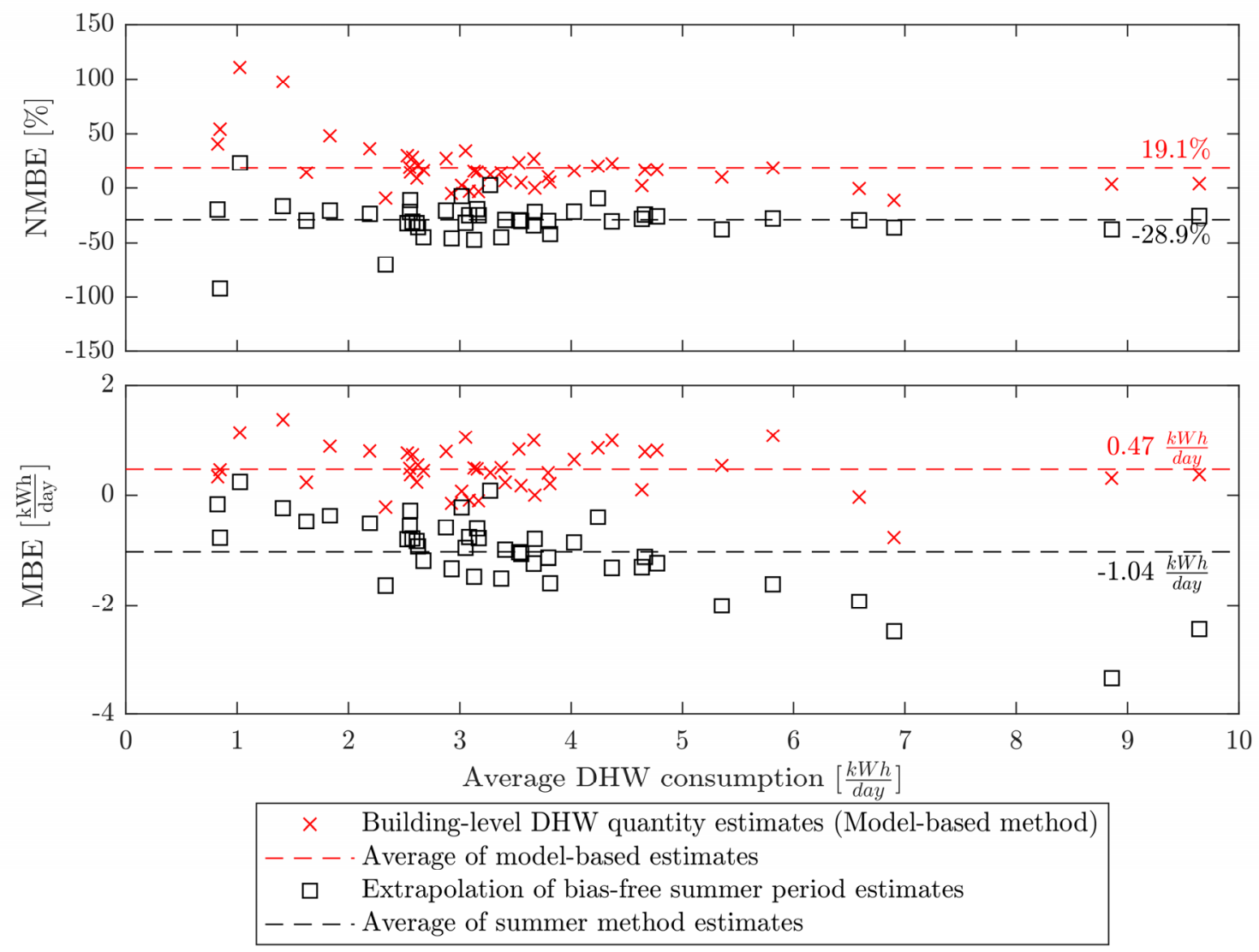

Fig. 6. Bias of the estimated DHW consumption for the 44 case buildings depicted in relative and absolute terms. The estimates of the model-based method are compared to the measured DHW consumption during summertime.

while the relative errors are easily relatable and comparable to other studies, they also tend to distort the results of especially buildings with low DHW consumption.

The results indicate that the proposed model-based method overestimate consumption by $0.47 \mathrm{kWh}$ per day or on average $13.1 \%$ in relative terms. This number is not to be confused with the $19.1 \%$ depicted in the upper part of the figure, which is a simple average of the relative error across the 44 buildings. The latter is therefore skewed by the large percentage-wise errors that are seen in buildings with low consumption.

Compared to the summertime measurements, which is the theoretical reference point for the summer methods, the mean bias error (MBE) of the estimates from the model-based method is less than $50 \%$ of the summer method bias, but with opposite sign. Although more validation data is needed to test the generality of these results, the larger bias of the summertime method is a clear example of the challenges associated with extrapolating summertime consumption levels to the rest of the year.

\section{Discussion}

There are several topics related to the analysis presented in this paper that should be further investigated in future work. The validation dataset used in this study comprised 44 similar buildings, but more and/or larger validation datasets are needed to further validate the method and to investigate its performance on other types and vintages of buildings. Furthermore, it is also necessary to investigate how the user-specified priors may have affected the results, and whether there are methods to avoid having these priors and still deliver similar or even better estimates of DHW consumption.

Finally, as identified in [19] and further indicated by the underestimation of the summer method depicted in Fig. 6, DHW consumption tends to feature a seasonal trend with increased consumption during wintertime. Future work should therefore aim at investigating how such a seasonal trend could be incorporated in the modelbased method and to which extent this could improve the estimates of DHW consumption.

\section{Conclusion}

In this paper we validated a model-based method for disaggregating the space heating and domestic hot water components of combined district heating measurements from remotely read smart meters. Consumption data from 44 terraced single-family houses that included separate measurements of domestic hot water usage were used to investigate and further develop the accuracy of the modelbased method. The method produces an overall estimate of the annual consumption levels, as well as a series of tapping profiles that describe the DHW consumption patterns in each given building. The results indicate that the method is capable of estimating the average weekprofile of the 44 case buildings with an MAE of $1.12 \frac{\mathrm{kWh}}{\mathrm{h}}$ and MAPE of $17.2 \%$. The results also shows that the 
method tended to overestimate the DHW consumption in the case buildings by approximately $0.5 \mathrm{kWh}$ per day. As a reference, the alternative approach of extrapolating summertime consumption to the full year led to an underestimation of approximately $1 \mathrm{kWh}$ per day for the case buildings.

The authors acknowledge the support received from the project 'Local heating concepts' supported by EUDP (Project number: 64017-0019), the project 'Data-driven demand side management in district heating' financed by the Danish Innovation Fund (Reference: 8119-00005B) and the READY project financed by the 7th EU Framework Program (Reference: 609127).

\section{References}

[1] European Parliament. DIRECTIVE (EU) 2018/2002 on Energy Efficiency

2018;2018:210-30.

[2] Yang S, Wan MP, Chen W, Ng BF, Zhai D. An adaptive robust model predictive control for indoor climate optimization and uncertainties handling in buildings. Build Environ 2019. https://doi.org/10.1016/j.buildenv.2019.106326.

[3] Cigler J, Prívara S, Váňa Z, Žáčeková E, Ferk1 L. Optimization of predicted mean vote index within model predictive control framework: Computationally tractable solution. Energy Build 2012;52:39-49. https://doi.org/10.1016/j.enbuild.2012.05.022.

[4] Burzynski R, Crane M, Yao R, Becerra VM. Space heating and hot water demand analysis of dwellings connected to district heating scheme in UK. J Cent South Univ Technol (English Ed 2012;19:1629-38. https://doi.org/10.1007/s11771-012-1186-z.

[5] Marszal-Pomianowska A, Zhang C, Pomianowski M, Heiselberg P, Gram-Hanssen K, Rhiger Hansen A. Simple methodology to estimate the mean hourly and the daily profiles of domestic hot water demand from hourly total heating readings. Energy Build 2019;184:53-64. https://doi.org/10.1016/j.enbuild.2018.11.035.

[6] Yliniemi K, Delsing J, van Deventer J. Experimental verification of a method for estimating energy for domestic hot water production in a 2-stage district heating substation. Energy Build 2009;41:169-74.

[7] Bacher P, de Saint-Aubain PA, Christiansen LE, Madsen H. Non-parametric method for separating domestic hot water heating spikes and space heating. Energy Build 2016;130:107-12. https://doi.org/10.1016/j.enbuild.2016.08.037.

[8] Fuentes E, Arce L, Salom J. A review of domestic hot water consumption profiles for application in systems and buildings energy performance analysis. Renew Sustain Energy Rev 2018;81:1530-47.

[9] Hedegaard RE, Kristensen MH, Pedersen TH, Brun A, Petersen S. Bottom-up modelling methodology for urban-scale analysis of residential space heating demand response. Appl Energy 2019;242:181-204. https://doi.org/10.1016/j.apenergy.2019.03.063.

[10] Bacher P, Madsen H. Identifying suitable models for the heat dynamics of buildings. Energy Build 2011;43:1511-22. https://doi.org/10.1016/j.enbuild.2011.02.005.

[11] Andersen KK, Madsen H, Hansen LH.

Modelling the heat dynamics of a building using stochastic differential equations. Energy Build 2000;31:13-24. https://doi.org/10.1016/S03787788(98)00069-3.

[12] Wang Z, Chen Y, Li Y. Development of RC model for thermal dynamic analysis of buildings through model structure simplification. Energy Build 2019;195:51-67. https://doi.org/10.1016/j.enbuild.2019.04.042.

[13] Hedegaard RE, Petersen S. Evaluation of GreyBox Model Parameter Estimates Intended for Thermal Characterization of Buildings. Energy Procedia, vol. 132, 2017.

https://doi.org/10.1016/j.egypro.2017.09.692.

[14] Reynders G, Diriken J, Saelens D. Quality of grey-box models and identified parameters as function of the accuracy of input and observation signals. Energy Build 2014;82:263-74. https://doi.org/10.1016/j.enbuild.2014.07.025.

[15] Harb H, Boyanov N, Hernandez L, Streblow R, Müller D. Development and validation of greybox models for forecasting the thermal response of occupied buildings. Energy Build 2016;117:199-207. https://doi.org/10.1016/j.enbuild.2016.02.021.

[16] Halvgaard R, Poulsen NK, Madsen H, Jørgensen JB. Economic Model Predictive Control for building climate control in a Smart Grid. 2012 IEEE PES Innov Smart Grid Technol ISGT 2012 2012:1-6. https://doi.org/10.1109/ISGT.2012.6175631.

[17] Dahl Knudsen M, Petersen S. Demand response potential of model predictive control of space heating based on price and carbon dioxide intensity signals. Energy Build 2016;125:196204.

https://doi.org/10.1016/j.enbuild.2016.04.053.

[18] Pedersen TH, Hedegaard RE, Petersen S. Space heating demand response potential of retrofitted residential apartment blocks. Energy Build 2017;141.

[19] Parker DS. Research highlights from a large scale residential monitoring study in a hot climate. Energy Build 2003.

[20] Energy Monitoring Company. Measurement of Domestic Hot Water Consumption in Dwellings 2008.

[21] Statens byggeforsknings institut (SBI). SBi 2009:10 Varmt Brugsvand. 2009.

[22] Pääkkönen H, Hannula U, Lehikoinen H, Ollila I. How Europeans spend their time Everyday life of women and men. 2004. 